\title{
23. STABLE ISOTOPE STRATIGRAPHY AND AMINO-ACID EPIMERIZATION FOR THE LAST 2.4 M.Y. AT SITE 610, HOLES 610 AND $610 A^{1}$
}

\author{
Eystein Jansen and Hans Petter Sejrup, Department of Geology, University of Bergen, Norway²
}

\begin{abstract}
A record of carbon and oxygen isotopes in benthic and planktonic foraminifers has been obtained from the interval corresponding to the last $2.4 \mathrm{~m}$.y. of Site 610 , Holes 610 and $610 \mathrm{~A}$, with a sample resolution of about $30 \mathrm{kyr}$. The record from the late Quaternary $(<0.9 \mathrm{Ma})$ shows large amplitudes and high frequencies in oxygen isotopic variation. Prior to $0.9 \mathrm{Ma}$ the isotopic variability record is reduced in amplitude (but not in frequency) compared with the late Quaternary, suggesting lower ice-volume and climatic fluctuations, and higher average eustatic sea level.

Left-coiling (L, polar) Neogloboquadrina pachyderma were not found in samples between 1.0 and $2.2 \mathrm{Ma}$, indicating less influence of polar front migrations in the Northeast Atlantic. Both polar planktonic faunas and larger isotope fluctuations reappear in the lowermost samples ( 2.3 to $2.4 \mathrm{Ma}$ ), pointing toward a period of larger climatic variability in the late Pliocene than in the early Quaternary.

The variation in benthic $\delta^{13} \mathrm{C}$ and hence in deep-water $\delta^{13} \mathrm{C}$ seems to have been constant through the analyzed section, reflecting a stable variability in the production of North Atlantic Deep Water (NADW) and possibly in Norwegian-Greenland Sea Overflow. Preliminary analyses of amino-acid epimerization in $N$. pachyderma (L) indicate a constant rate of epimerization to approximately $0.3 \mathrm{Ma}$. Beneath this level the average epimerization rate is much reduced.
\end{abstract}

\section{INTRODUCTION}

The introduction of the hydraulic piston corer (HPC) has offered new possibilities for detailed studies of undisturbed, unconsolidated sediments covering large parts of the Neogene, and has provided a potential for studying cores with high deposition rates far beyond the limits of conventional piston coring. Site 610 is located on the Feni Drift (Fig. 1) at $2417 \mathrm{~m}$ depth. The different HPC-drilled holes at this site constitute an almost complete section, down to $200 \mathrm{~m}$ sub-bottom depth, revealing a high sedimentation rate of approximately $5.2 \mathrm{~cm} /$ kyr.

The main objective of this study was to provide a benthic and planktonic $\delta^{13} \mathrm{C}$ and $\delta^{18} \mathrm{O}$ record for the upper $122.5 \mathrm{~m}$ at this site in order to elucidate some of the main trends in isotopic variability within the cores. This record should serve as a pilot study to test the feasibility of obtaining detailed isotopic records in these sediments. Our sampling interval of $1.5 \mathrm{~m}$ (approximately $30 \mathrm{kyr}$.) should be sufficient to determine the primary amplitudes in climatic variability, and to test whether the Quaternary climatic oscillations have been variable or constant in amplitude, and if there were intervals when the overall pattern of climatic variation changed. Studies of DSDP Hole 502B in the Caribbean indicate higher-amplitude climatic fluctuations within the late Quaternary (Brunhes Epoch) than in the early Quaternary (Prell, 1982). The carbon isotopic composition of deep-sea benthic foraminifers may be used as an indicator of rates of deep-water renewal, and in our case may provide infor-

\footnotetext{
${ }^{1}$ Ruddiman, W. F., Kidd, R. B., Thomas, E., et al., Init. Repts. DSDP, 94: Washington (U.S. Govt. Printing Office).

2 Address: Dept. of Geology, Section B, University of Bergen, Allégaten 41, N-5000 Bergen, Norway.
}

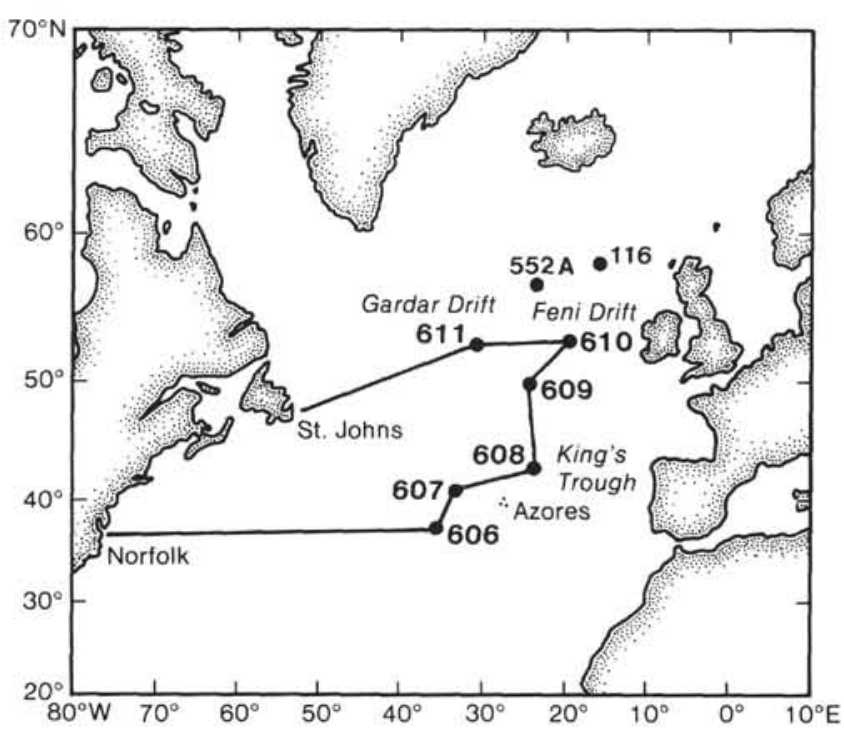

Figure 1. Location of Leg 94 sites and DSDP Holes 552A and 116.

mation on variations in the production of North Atlantic Deep Water (NADW) and possibly Norwegian-Greenland Sea Overflow. We have also analyzed planktonic foraminifers in order to study differences in deep-water and surface isotopic response.

In addition, we report here the first results from an ongoing study of the diagenesis in the protein remains in foraminifers from Site 610, Holes 610 and 610A. The major objectives of this study are to: (1) evaluate the potential of using amino-acid diagenesis as a geochronological tool in deep-sea cores, and to determine what precision level this method has; (2) investigate how isoleucine epimerization reaction rates for different species relate to each other, and whether they change in time; (3) evaluate the influence of geothermal heat flow on 
the results by comparison with results from cores with slower sedimentation rates (King and Neville, 1977; Bada and Man, 1980; Müller, 1984); and (4) investigate the possibility of using amino-acid data to evaluate possible differences in bottom-water temperatures in different deepsea basins.

So far only the epimerization of L-isoleucine to D-alloisoleucine in the planktonic foraminifer Neogloboquadrina pachyderma left-coiling $(\mathrm{L})$ has been investigated.

\section{METHODS}

\section{Stable Isotope Analysis}

Samples were wet-sieved and dried at $30^{\circ} \mathrm{C}$. Monospecific samples were picked from the $>125-\mu \mathrm{m}$ fraction, cleaned ultrasonically in methanol, and roasted for $40 \mathrm{~min}$. in vacuo at $380^{\circ} \mathrm{C}$. $\mathrm{CO}_{2}$ for analysis was extracted by reaction with $100 \%$ orthophosphoric acid at $50^{\circ} \mathrm{C}$ on-line to the mass-spectrometer and cleaned from water by a cold trap at $-95^{\circ} \mathrm{C}$.

The isotopic measurements were performed on an automated Finnigan MAT 251 mass spectrometer. All data are presented with respect to the PDB standard. Calibration to PDB is produced through comparison with Cambridge, Lamont, and Copenhagen standards as well as standards supplied by the National Bureau of Standards (NBS 18 and NBS 19). The analytical precision is $\pm 0.1 \%$ for $\delta^{18} \mathrm{O}$ and $\pm 0.07 \%$ for $\delta^{13} \mathrm{C}$. Replicate analyses of the same samples were performed on a few levels that contained enough benthic carbonate, and gave a reproducibility within $0.3 \%$ for $\delta^{18} \mathrm{O}$ and $\delta^{13} \mathrm{C}$.

\section{Amino-Acid Analysis}

Monospecific samples of $N$. pachyderma $(\mathrm{L})$ were picked from the $>125-\mu \mathrm{m}$ fraction. Each sample consisted of about 100 well-preserved specimens. The samples were repeatedly cleaned with purified water in an ultrasonic bath following the procedure described by Miller et al. (1983). After the tests were dissolved in $7 \mathrm{~N} \mathrm{HCl}$, using Norleucine as an internal standard, the samples were hydrolyzed at $110^{\circ} \mathrm{C}$ for $22 \mathrm{hr}$., and subsequently dried under vacuum, rehydrated with a $\mathrm{pH} 2$ solution, and analyzed in an automatic ion-exchange HPLC amino-acid analyzer. The D-alloisoleucine/L-isoleucine ratios presented in this chapter represent the ratios between peak heights as determined by a HewlettPackard computing integrator. The extent of epimerization, given as the ratio between D-alloisoleucine and L-isoleucine (alle/Ile), is close to 0.01 in a modern sample and reaches an equilibrium at a value of circa 1.4 in Miocene samples from deep-sea cores (King and Neville, 1977; Müller, 1984).

During the same period when the samples from Leg 94 were analyzed, the Bergen Amino Acid Geochronology Laboratory (BAL) analyzed the interlaboratory calibration standards presented by Wehmiller (1984) with the following alle/Ile ratios in the total fraction: 81-ICAA: $0.167 \pm 0.003$; 81 -ICA-B: $0.540 \pm 0.016 ; 81$-ICA-C: $1.20 \pm 0.048$.

\section{ISOTOPE RESULTS}

Both benthic and planktonic foraminiferal assemblages show large downcore variations in faunal composition. Consequently it is not possible to base the isotope stratigraphy on a single species; therefore we analyzed a range of different benthic species in order to produce a complete record. Because most of these species have been reported to show constant oxygen isotopic disequilibria in earlier studies, it is possible to adjust the values for species-specific departures from oxygen isotopic equilibrium. The factors we have applied are summarized in Table 1. Although there are widely accepted correction factors for carbon isotope values from the common benthic species Uvigerina peregrina and Cibicides wuellerstorfi, there is a rather large scatter in the values proposed for the other species we have used (Shackleton, 1974; Belanger et al., 1981), which imposes some uncertainties on the
Table 1. Correction factors applied for benthic foraminifers at Site 610 .

\begin{tabular}{|c|c|c|c|}
\hline Species & $\delta^{18} \mathrm{O}$ & ${ }_{\delta}^{13} \mathrm{C}$ & Reference \\
\hline Cibicides wuellerstorfi & +0.64 & 0.00 & $\begin{array}{l}\text { Shackleton and Opdyke, 1973; } \\
\text { Duplessy et al., } 1984\end{array}$ \\
\hline $\left.\begin{array}{l}\text { Melonis barleeanum } \\
\text { Melonis pompilioides }\end{array}\right\}$ & +0.40 & +0.36 & $\begin{array}{l}\text { Graham et al., 1981; } \\
\text { Woodruff et al., } 1980\end{array}$ \\
\hline Uvigerina peregrina & 0.00 & +0.90 & $\begin{array}{l}\text { Shackleton, 1974; Duplessy et } \\
\text { al., } 1984\end{array}$ \\
\hline Oridorsalis tener & +0.36 & +1.17 & $\begin{array}{l}\text { CLIMAP Project Members, } \\
\text { 1984; } \\
\text { Graham et al., } 1981\end{array}$ \\
\hline Pyrgo murrhina & 0.00 & 0.00 & $\begin{array}{l}\text { Shackleton, 1974; Ganssen, } \\
\text { 1983; } \\
\text { Graham et al., } 1981\end{array}$ \\
\hline Cassidulina teretis & +0.30 & +1.17 & Jansen, preliminary results \\
\hline
\end{tabular}

carbon isotope record and may account for some of the large peaks. Most notably the values for Pyrgo murrhi$n a$ and Melonis barleeanum may be unreliable.

For the planktonic record we usually analyzed $N$. pachyderma $(\mathrm{L})$, and in samples where this species was missing we analyzed $N$. pachyderma right-coiling (R) and Globigerina bulloides. With some exceptions this provided us with a complete planktonic record.

We have tabulated the isotope results in Tables 2 through 5 and have given both the measured values and the supposed isotopic equilibrium values for benthic foraminifers. Most samples contained enough specimens of one benthic species to make an analysis. In some samples we were able to perform analyses on more than one species and found them generally to reproduce the known differences in departures from isotopic equilibrium. In some samples the amount of benthic carbonate available was too small to enable analysis. This situation was most serious in Core 610A-12. Although the results demonstrate the feasibility of producing a detailed isotope stratigraphy, it might prove difficult to provide a complete record with the desired sampling density in some parts of the hole.

Planktonic foraminifers were available in large numbers in all samples. The primary difficulty with the planktonic record is the large variation in the planktonic assemblages, making it impossible to make a single species record. The three species we have used were normally present in adequate amounts either alone or together; nevertheless it was not possible to obtain measurable numbers of specimens of these species in some samples. Through large parts of the section we were unable to find $N$. pachyderma (L) (Fig. 2). In the samples where the normal, coarse-textured and involute $N$. pachyder$m a(\mathrm{~L})$ was absent it was possible to identify smaller foraminifers resembling $N$. pachyderma. These do not occur in polar assemblages, and analyses of their isotopic composition display values substantially lighter in $\delta^{18} \mathrm{O}$ than expected values for $N$. pachyderma. The isotope values are lighter than those of both $N$. pachyderma $(\mathrm{R})$ and $G$. bulloides from the same samples. We thus con- 
Table 2. Oxygen and carbon isotopic composition of benthic foraminifers at Hole 610A.

\begin{tabular}{|c|c|c|c|c|c|c|c|}
\hline $\begin{array}{l}\text { Core- } \\
\text { section }\end{array}$ & $\begin{array}{c}\text { Sample } \\
\text { interval } \\
(\mathrm{cm})\end{array}$ & $\begin{array}{l}\text { Sub-bottom } \\
\text { depth } \\
\text { (m) }\end{array}$ & Species & $\delta^{18} \mathrm{O}_{\mathrm{PDB}}$ & $\begin{array}{c}\delta^{18} \mathrm{O}_{\mathrm{PDB}} \\
\text { (corr. values) }\end{array}$ & $\delta^{13} \mathrm{C}_{\mathrm{PDB}}$ & $\begin{array}{c}\delta^{13} \mathrm{C}_{\mathrm{PDB}} \\
\text { (corr. values) }\end{array}$ \\
\hline $1-1$ & $44-46$ & 0.45 & C. wuellerstorfi & +2.61 & +3.25 & +0.83 & +0.83 \\
\hline $1-2$ & 44-46 & 1.95 & P. murrhina & +4.45 & +4.45 & +1.14 & +1.14 \\
\hline $1-3$ & $44-46$ & 3.45 & C. wuellerstorfi & +3.60 & +4.24 & +0.77 & +0.77 \\
\hline $1-4$ & $54-56$ & 5.05 & C. wuellerstorfi & +3.38 & +4.02 & +0.53 & +0.53 \\
\hline $1-5$ & $44-46$ & 6.45 & C. wuellerstorfi & +3.11 & +3.75 & +0.92 & +0.92 \\
\hline $1-6$ & $44-46$ & 7.95 & C. wuellerstorfi & +3.32 & +3.96 & +0.65 & +0.65 \\
\hline $2-1$ & $44-46$ & 9.85 & C. wuellerstorfi & +3.61 & +4.25 & +0.82 & +0.82 \\
\hline $2-2$ & $54-56$ & 11.05 & M. barleeanum & +4.03 & +4.43 & -0.06 & +0.30 \\
\hline $2-6$ & $64-66$ & 17.15 & C. wuellerstorfi & +3.32 & +3.96 & +1.06 & +1.06 \\
\hline $3-1$ & $44-46$ & 19.05 & C. wuellerstorfi & +3.57 & +4.20 & +0.11 & +0.11 \\
\hline $3-2$ & $44-46$ & 20.55 & C. wuellerstorfi & +2.93 & +3.57 & +0.77 & +0.77 \\
\hline $3-3$ & $44-46$ & 22.05 & C. wuellerstorfi & +2.61 & +3.25 & +0.90 & +0.90 \\
\hline 3-4 & $44-46$ & 23.55 & M. barleeanum & +4.37 & +4.77 & -0.43 & -0.07 \\
\hline $3-5$ & $44-46$ & 25.05 & $U$. peregrina & +4.27 & +4.27 & -0.50 & +0.40 \\
\hline 3-6 & $44-46$ & 26.55 & $U$. peregrina & +3.57 & +3.57 & +0.22 & +1.12 \\
\hline $4-2$ & $44-46$ & 30.15 & $U$. peregrina & +3.83 & +3.83 & -0.35 & +0.55 \\
\hline 4-3 & 44-46 & 31.65 & C. wuellerstorfi & +3.34 & +3.98 & +0.48 & +0.48 \\
\hline $4-4$ & $44-46$ & 33.15 & M. barleeanum & +2.96 & +3.36 & -0.53 & -0.17 \\
\hline $4-5$ & $44-46$ & 34.65 & M. barleeanum & +3.47 & +3.87 & +0.20 & +0.16 \\
\hline $4-6$ & $44-46$ & 36.15 & C. wuellerstorfi & +4.09 & +4.73 & +0.36 & +0.36 \\
\hline $5-2$ & $44-46$ & 39.75 & C. wuellerstorfi & +3.27 & +3.91 & +0.83 & +0.83 \\
\hline $5-3$ & $44-46$ & 41.25 & M. barleeanum & +3.74 & +4.14 & -0.87 & -0.51 \\
\hline $5-4$ & $44-46$ & 42.75 & C. wuellerstorfi & +2.97 & +3.61 & -0.16 & -0.16 \\
\hline $5-5$ & 44-46 & 44.25 & M. barleeanum/pompilioides & +2.84 & +3.24 & -0.29 & +0.07 \\
\hline $5-6$ & $44-46$ & 45.75 & M. barleeanum/pompilioides & +3.23 & +3.67 & -0.58 & -0.22 \\
\hline $6-1$ & $44-46$ & 47.85 & M. barleeanum/pompilioides & +3.32 & +3.72 & -0.42 & -0.06 \\
\hline $6-2$ & $44-46$ & 49.35 & P. murrhina & +4.51 & +4.51 & -0.80 & -0.80 \\
\hline $6-2$ & $44-46$ & 49.35 & M. pompilioides & +3.65 & +4.05 & -1.30 & -1.11 \\
\hline $6-4$ & $44-46$ & 52.35 & C. wuellerstorfi & +3.03 & +3.67 & +0.02 & +0.02 \\
\hline $6-5$ & $44-46$ & 53.85 & C. wuellerstorfi & +3.13 & +3.77 & +0.38 & +0.38 \\
\hline $6-6$ & $44-46$ & 55.35 & C. wuellerstorfi & +3.60 & +4.24 & +0.70 & +0.70 \\
\hline $7-2$ & $44-46$ & 58.95 & M. pompilioides & +3.11 & +3.51 & +0.38 & +0.74 \\
\hline $7-3$ & $44-46$ & 60.45 & O. tener & +3.83 & +4.19 & -0.63 & +0.54 \\
\hline $7-5$ & $44-46$ & 63.45 & C. wuellerstorfi & +3.05 & +3.69 & +0.41 & +0.41 \\
\hline $7-6$ & $44-46$ & 64.95 & Cibicides sp. & +3.38 & +4.02 & -0.49 & -0.49 \\
\hline $8-1$ & $44-46$ & 67.05 & C. wuellerstorfi & +2.68 & +3.32 & +0.97 & +0.97 \\
\hline $8-2$ & $44-46$ & 68.55 & C. wuellerstorfi & +2.94 & +3.58 & +0.40 & +0.40 \\
\hline $8-3$ & $44-46$ & 70.05 & $U$. peregrina & +3.86 & +3.86 & -0.58 & +0.32 \\
\hline $8-5$ & $44-46$ & 73.05 & C. wuellerstorfi & +2.67 & +3.31 & +0.30 & +0.30 \\
\hline $8-6$ & $44-46$ & 74.55 & P. murrhina & +2.71 & +2.71 & +0.29 & +0.29 \\
\hline $9-1$ & $44-46$ & 76.65 & C. wuellerstorfi & +2.19 & +2.83 & +0.63 & +0.63 \\
\hline $9-2$ & $44-46$ & 78.15 & C. wuellerstorfi & +2.45 & +3.09 & +0.83 & +0.83 \\
\hline $9-3$ & $44-46$ & 79.65 & C. wuellerstorfi & +3.43 & +4.07 & +0.64 & +0.64 \\
\hline $9-4$ & $44-46$ & 81.15 & C. wuellerstorfi & +3.33 & +3.97 & +0.42 & +0.42 \\
\hline $9-5$ & $44-46$ & 82.65 & C. wuellerstorfi & +2.71 & +3.35 & +0.64 & +0.64 \\
\hline $9-6$ & $44-46$ & 84.15 & P. murrhina & +4.08 & +4.08 & +0.56 & +0.56 \\
\hline $10-1$ & $44-46$ & 86.25 & C. wuellerstorfi & +2.50 & +3.14 & +1.09 & +1.09 \\
\hline $10-2$ & $44-46$ & 87.75 & M. pompilioides & +3.49 & +3.89 & -0.14 & +0.22 \\
\hline $10-3$ & $44-46$ & 89.25 & C. wuellerstorfi & +3.06 & +3.70 & +0.57 & +0.57 \\
\hline $10-4$ & $44-46$ & 90.75 & P. murrhina & +3.26 & +3.26 & +0.21 & +0.21 \\
\hline $10-6$ & $44-46$ & 93.75 & $U$. peregrina & +3.26 & +3.26 & -0.02 & +0.88 \\
\hline $11-2$ & $44-46$ & 97.35 & C. wuellerstorfi & +2.60 & +3.24 & +0.11 & +0.11 \\
\hline $11-4$ & $44-46$ & 100.35 & C. wuellerstorfi & +2.48 & +3.12 & +1.23 & +1.23 \\
\hline $11-5$ & $44-46$ & 101.85 & $U$. peregrina & +2.81 & +2.81 & +0.11 & +1.01 \\
\hline $11-6$ & $44-46$ & 103.35 & P. murrhina & +3.14 & +3.14 & +0.67 & +0.67 \\
\hline $12-1$ & $44-46$ & 105.45 & M. barleeanum & +3.01 & +3.41 & -1.04 & -0.68 \\
\hline $12-2$ & $44-46$ & 106.95 & $U$. peregrina & +3.31 & +3.31 & -0.16 & +0.74 \\
\hline $12-4$ & $44-46$ & 109.95 & $U$. peregrina & +3.18 & +3.18 & +0.24 & +1.14 \\
\hline $13-3$ & $44-46$ & 118.05 & C. wuellerstorfi & +2.78 & +3.43 & +0.52 & +0.52 \\
\hline $13-4$ & $44-46$ & 119.55 & O. tener & +4.48 & +4.84 & -0.66 & +0.51 \\
\hline $13-5$ & $44-46$ & 121.05 & C. wuellerstorfi & +2.57 & +3.21 & +0.89 & +0.89 \\
\hline $13-6$ & $44-46$ & 122.55 & M. barleeanum & +2.98 & +3.38 & -0.26 & +0.07 \\
\hline
\end{tabular}

clude that these small variants should not be considered as $N$. pachyderma, at least not with regard to their isotopic composition. Right-coiling $N$. pachyderma are on the average lighter in $\delta^{13} \mathrm{C}$ by $0.35 \%$ and in $\delta^{18} \mathrm{O}$ by $0.45 \%$ than $G$. bulloides, although the sample-to-sample difference may vary markedly (Fig. 2) (Tables 3 and 5). This is in accordance with the isotopic patterns for these species revealed by a study of their recent distribu- tion in the Norwegian Sea (T. Johannessen, personal communication, 1984).

Coring disturbances are present in some of the upper cores from Hole 610A. A number of samples from Hole 610 were thus analyzed in order to fill in gaps in the sediment recovery of Hole $610 \mathrm{~A}$. A summary of the intervals that were reported to show severe coring disturbance is presented in Figure 2. Some of the uppermost sedi- 
Table 3. Oxygen and carbon isotopic composition of planktonic foraminifers at Hole 610A.

\begin{tabular}{|c|c|c|c|c|c|}
\hline $\begin{array}{l}\text { Core- } \\
\text { section }\end{array}$ & $\begin{array}{c}\text { Sample } \\
\text { interval } \\
\text { (cm) }\end{array}$ & $\begin{array}{l}\text { Sub-bottom } \\
\text { depth } \\
\text { (m) }\end{array}$ & Species & ${ }^{18} \mathrm{O}_{\mathrm{PDB}}$ & $\delta^{13} C_{\text {PDB }}$ \\
\hline $1-1$ & $44-46$ & 0.45 & N. pachyderma $(\mathrm{R})$ & +0.95 & +0.29 \\
\hline $1-2$ & $44-46$ & 1.95 & N. pachyderma $(\mathrm{L})$ & +3.70 & -0.42 \\
\hline $1-3$ & $44-46$ & 3.45 & N. pachyderma (L) & +3.44 & -0.63 \\
\hline 1.4 & $44-46$ & 5.05 & N. pachyderma (L) & +2.79 & -0.95 \\
\hline $1-5$ & $44-46$ & 6.45 & N. pachyderma (L) & +2.46 & +0.61 \\
\hline $1-6$ & $44-46$ & 7.95 & N. pachyderma (L) & +2.86 & +0.25 \\
\hline $2-1$ & $84-86$ & 9.85 & N. pachyderma (L) & +2.95 & -0.18 \\
\hline $2-2$ & $54-56$ & 11.55 & N. pachyderma (L) & +3.68 & -0.40 \\
\hline $2-6$ & $64-66$ & 17.15 & N. pachyderma (L) & +2.79 & -0.13 \\
\hline $3-1$ & $44-46$ & 19.05 & N. pachyderma (L) & +3.38 & -0.62 \\
\hline $3-2$ & $44-46$ & 20.55 & N. pachyderma (L) & +2.55 & +0.03 \\
\hline 3-3 & $44-46$ & 22.05 & N. pachyderma $(\mathrm{R})$ & +0.81 & +0.26 \\
\hline $3-4$ & $44-46$ & 23.55 & N. pachyderma (L) & +3.46 & -0.17 \\
\hline $3-5$ & $44-46$ & 25.05 & N. pachyderma (L) & +3.30 & -0.41 \\
\hline $3-6$ & $44-46$ & 26.55 & N. pachyderma (L) & +2.00 & +0.72 \\
\hline $4-2$ & $44-46$ & 30.15 & N. pachyderma (R) & +1.50 & +0.02 \\
\hline 4-3 & $44-46$ & 31.65 & N. pachyderma (L) & +2.84 & -0.72 \\
\hline 4-4 & 44-46 & 33.15 & N. pachyderma (R) & +1.36 & -0.55 \\
\hline $4-5$ & $44-46$ & 34.65 & N. pachyderma (L) & +2.67 & -0.15 \\
\hline $4-6$ & $44-46$ & 36.15 & N. pachyderma (L) & +3.48 & -0.13 \\
\hline $5-3$ & $44-46$ & 41.25 & N. pachyderma (L) & +2.66 & -1.09 \\
\hline $5-4$ & $44-46$ & 42.75 & N. pachyderma (L) & +2.34 & -0.50 \\
\hline $5-6$ & $44-46$ & 45.75 & N. pachyderma (L) & +1.96 & -0.64 \\
\hline $6-1$ & $44-46$ & 47.85 & N. pachyderma $(\mathrm{L})$ & +2.39 & -0.65 \\
\hline $6-2$ & $44-46$ & 49.35 & N. pachyderma $(\mathrm{L})$ & +2.70 & -1.26 \\
\hline $6-4$ & $44-46$ & 52.35 & N. pachyderma (L) & +2.54 & +0.12 \\
\hline $6-5$ & $44-46$ & 53.85 & N. pachyderma (L) & +2.55 & +0.28 \\
\hline $6-6$ & $44-46$ & 55.35 & N. pachyderma (L) & +2.41 & +0.09 \\
\hline 7.2 & $44-46$ & 58.95 & N. pachyderma (L) & +1.99 & -0.28 \\
\hline $7-3$ & $44-46$ & 60.45 & N. pachyderma (L) & +1.98 & -0.53 \\
\hline $7-4$ & $44-46$ & 61.95 & N. pachyderma (R) & +1.19 & -0.41 \\
\hline $7-6$ & $44-46$ & 64.95 & N. pachyderma (R) & +1.05 & +0.20 \\
\hline $8-1$ & $44-46$ & 67.05 & N. pachyderma $(\mathrm{R})$ & +0.88 & +0.01 \\
\hline $8-2$ & $44-46$ & 68.55 & G. bulloides & +1.26 & -0.75 \\
\hline $8-3$ & $44-46$ & 70.05 & N. pachyderma $(\mathrm{R})$ & +1.17 & -0.98 \\
\hline $8-4$ & $44-46$ & 71.55 & N. pachyderma (R) & +0.36 & -0.47 \\
\hline $8-4$ & $44-46$ & 71.55 & G. bulloides & +0.96 & -0.57 \\
\hline $8-5$ & $44-46$ & 73.05 & G. bulloides & +1.26 & -0.44 \\
\hline $8-6$ & $44-46$ & 74.55 & G. bulloides & +1.75 & -0.30 \\
\hline $9-1$ & $44-46$ & 76.65 & N. pachyderma $(\mathrm{R})$ & +0.65 & -0.43 \\
\hline $9-2$ & $44-46$ & 78.15 & G. bulloides & +1.20 & +0.26 \\
\hline $9-3$ & $44-46$ & 79.65 & N. pachyderma $(\mathrm{R})$ & +1.66 & -0.46 \\
\hline $9-3$ & $44-46$ & 79.65 & G. bulloides & +1.76 & -0.26 \\
\hline $9-5$ & $44-46$ & 82.65 & G. bulloides & +1.86 & +0.24 \\
\hline $9-6$ & $44-46$ & 84.15 & G. bulloides & +2.52 & +0.26 \\
\hline $10-1$ & $44-46$ & 86.25 & G. bulloides & +1.95 & +0.41 \\
\hline $10-2$ & $44-46$ & 87.25 & G. bulloides & +1.95 & +0.32 \\
\hline $10-2$ & 44-46 & 87.75 & N. pachyderma (R) & +1.24 & -0.97 \\
\hline $10-3$ & $44-46$ & 89.25 & N. pachyderma (R) & +1.51 & -1.01 \\
\hline $10-3$ & $44-46$ & 89.25 & G. bulloides & +1.70 & -0.69 \\
\hline $10-4$ & $44-46$ & 90.75 & N. pachyderma (R) & +1.36 & -1.03 \\
\hline $10-4$ & $44-46$ & 90.75 & G. bulloides & +1.88 & -0.39 \\
\hline $10-5$ & $44-46$ & 92.25 & N. pachyderma $(\mathrm{R})$ & +1.21 & -0.43 \\
\hline $10-5$ & $44-46$ & 92.25 & G. bulloides & +1.30 & -0.13 \\
\hline $10-6$ & $44-46$ & 93.75 & N. pachyderma (R) & +1.50 & -0.13 \\
\hline $11-2$ & $44-46$ & 97.35 & N. pachyderma $(\mathrm{R})$ & +1.42 & +0.21 \\
\hline $11-3$ & $44-46$ & 98.85 & N. pachyderma (R) & +1.04 & +0.03 \\
\hline $11-4$ & $44-46$ & 100.35 & G. bulloides & +1.80 & +0.30 \\
\hline $11-4$ & $44-46$ & 100.35 & N. pachyderma $(\mathrm{R})$ & +1.44 & +0.14 \\
\hline $11-5$ & $44-46$ & 101.85 & G. bulloides & +1.69 & +0.64 \\
\hline $11-6$ & $44-46$ & 103.35 & G. bulloides & +2.06 & +0.35 \\
\hline $12-1$ & $44-46$ & 105.45 & G. bulloides & +2.03 & -1.09 \\
\hline $12-2$ & $44-46$ & 106.95 & G. bulloides & +1.66 & -0.76 \\
\hline $12-3$ & $44-46$ & 108.45 & N. pachyderma $(\mathrm{R})$ & +1.39 & -0.24 \\
\hline $12-4$ & $44-46$ & 109.95 & N. pachyderma (R) & +1.09 & +0.19 \\
\hline $12-5$ & $44-46$ & 111.45 & N. pachyderma (R) & +1.09 & +0.32 \\
\hline $12-5$ & $44-46$ & 111.45 & G. bulloides & +1.68 & +0.03 \\
\hline $13-2$ & $44-46$ & 116.55 & G. bulloides & +1.74 & +0.41 \\
\hline $13-2$ & 44-46 & 116.55 & N. pachyderma $(\mathrm{R})$ & +0.81 & +0.04 \\
\hline $13-3$ & $44-46$ & 118.05 & N. pachyderma (R) & +1.30 & +0.39 \\
\hline $13-4$ & $44-46$ & 119.55 & N. pachyderma (L) & +2.87 & +0.87 \\
\hline $13-5$ & $44-46$ & 121.05 & N. pachyderma (L) & +2.05 & +1.25 \\
\hline $13-6$ & $44-46$ & 122.55 & N. pachyderma (L) & +2.60 & +0.46 \\
\hline
\end{tabular}

ments may have been lost during coring in Hole 610, as the top sample, taken at $0.45 \mathrm{~m}$ in this hole, shows glacial planktonic assemblages and isotope values, whereas the sample from the same level in Hole 610A shows Hol- ocene assemblages and isotope values. The Brunhes/Matuyama $(\mathrm{B} / \mathrm{M})$ magnetic boundary is somewhat shallower in Hole 610 than in 610A. These differences make it difficult to match precisely samples from the two holes in order to form a single record. Ruddiman, Cameron, and Clement (this volume) performed detailed visual correlations of the different offset holes at Site 610 . Their results indicate that corresponding levels in Holes 610 and $610 \mathrm{~A}$ are not offset by a constant factor downhole, but rather that correlative levels are found to be located with a difference in sub-bottom depths ranging between 25 and $100 \mathrm{~cm}$. In order to fit the data from Hole 610 into the record from Hole 610A we have used the visual correlations of Ruddiman et al., and the upper level of the Brunhes/Matuyama boundary. By this method the sub-bottom depths of the samples from Hole 610 are moved downward by the following factors (Figs. 2 and 4): $0-5 \mathrm{~m}-25 \mathrm{~cm} ; 5-15.5 \mathrm{~m}-60 \mathrm{~cm} ; 15.5-34.5 \mathrm{~m}-75$ $\mathrm{cm} ; 34.5-47 \mathrm{~m}-100 \mathrm{~cm}$. The problems of hole-to-hole correlations show that it is not an easy task to place a single sample in its right place. We thus note that the method we have used may have led to possible errors, and that the composite record we have obtained cannot be regarded as a continuous one. A correct placing of the data is dependent on matching of complete curves based on a much higher sampling density than the one used for this study.

\section{DISCUSSION}

\section{Oxygen Isotopes}

Oxygen isotopes in benthic foraminifers are believed to be rough measures of global ice volume (Shackleton, 1967; Shackleton and Opdyke, 1973). Studies by Duplessy et al. (1980) indicate that there might be a temperature overprint on benthic $\delta^{18} \mathrm{O}$ records from the Northeast Atlantic Basin, as a result of lower glacial deep-water temperatures. The benthic record and the record from $N$. pachyderma $(\mathrm{L})$ follow similar trends, reflecting the proposed deep habitat for $N$. pachyderma (L) (Kellogg et al., 1978; Duplessy et al., 1981). High-amplitude isotopic peaks are recorded throughout the Brunhes Epoch and down to approximately $0.9 \mathrm{Ma}$, just above the Jaramillo Event on the paleomagnetic time scale (Fig. 2). This reflects the large fluctuations in ice volume and climate known from numerous isotope records from the late Quaternary. The extreme difference between peak glacial and peak interglacial values is approximately $1.8 \%$, in good accordance with expected values in records with high sedimentation rates. We also note that ice-volume minima coincide with the absence of polar planktonic foraminiferal species, and thus with warm surface-water conditions.

According to our records, before $0.9 \mathrm{Ma}$ there was a continuation of high-frequency climatic variations, but the severity of these variations were markedly reduced in comparison with those of the late Quaternary. A summary of the mean isotopic values and variability (expressed as the standard deviation about the mean) is presented in Table 6 and Figure 3. The mean benthic oxygen isotope value is $0.4 \%$ lighter prior to $0.9 \mathrm{Ma}$ than 
Table 4. Oxygen and carbon isotopic composition of benthic foraminifers at Hole 610 .

\begin{tabular}{|c|c|c|c|c|c|c|c|}
\hline $\begin{array}{l}\text { Core- } \\
\text { section }\end{array}$ & $\begin{array}{c}\text { Sample } \\
\text { interval } \\
(\mathrm{cm})\end{array}$ & $\begin{array}{l}\text { Sub-bottom } \\
\text { depth } \\
\text { (m) }\end{array}$ & Species & ${ }^{18} \mathrm{O}_{\mathrm{PDB}}$ & $\begin{array}{c}\delta^{18} \mathrm{O}_{\mathrm{PDB}} \\
\text { (corr. values) }\end{array}$ & $\delta^{13} \mathrm{C}_{\mathrm{PDB}}$ & $\begin{array}{c}{ }^{\delta^{13}} \mathrm{C}_{\mathrm{PDB}} \\
\text { (corr. values) }\end{array}$ \\
\hline $1-1$ & $44-46$ & 0.45 & M. barleeanum & +3.89 & +4.29 & -0.16 & +0.20 \\
\hline $1-2$ & $44-46$ & 1.95 & $U$. peregrina & +4.45 & +4.45 & -0.24 & +0.66 \\
\hline $1-2$ & $44-46$ & 1.95 & C. wuellerstorfi & +3.83 & +4.47 & +0.97 & +0.97 \\
\hline $1-3$ & $44-46$ & 3.45 & C. wuellerstorfi & +3.64 & +4.29 & +0.47 & +0.47 \\
\hline $1-4$ & $44-46$ & 4.95 & C. wuellerstorfi & +3.70 & +4.34 & +1.03 & +1.03 \\
\hline $1-6$ & $44-46$ & 7.95 & C. teretis & +4.00 & +4.30 & -1.52 & +0.35 \\
\hline $2-1$ & $44-46$ & 10.05 & C. wuellerstorfi & +3.70 & +4.34 & +0.13 & +0.13 \\
\hline $2-1$ & $44-46$ & 10.05 & P. murrhina & +4.29 & +4.29 & +0.68 & +0.68 \\
\hline $2-2$ & $44-46$ & 11.55 & C. wuellerstorfi & +2.71 & +3.35 & +0.98 & +0.98 \\
\hline $2-3$ & $44-46$ & 13.05 & $U$. peregrina & +3.22 & +3.22 & -0.19 & +0.71 \\
\hline $2-3$ & $44-46$ & 13.05 & C. wuellerstorfi & +2.19 & +2.83 & +0.63 & +0.63 \\
\hline $2-3$ & $44-46$ & 13.05 & M. barleeanum & +2.59 & +2.99 & +0.37 & +0.73 \\
\hline $2-4$ & $44-46$ & 14.55 & $U$. peregrina & +4.42 & +4.42 & -0.57 & +0.33 \\
\hline $2-5$ & $44-46$ & 16.05 & $U$. peregrina & +4.05 & +4.05 & -0.34 & +0.56 \\
\hline $3-3$ & $24-26$ & 22.45 & C. wuellerstorfi & +2.33 & +2.97 & +1.06 & +1.06 \\
\hline $3-4$ & $44-46$ & 24.15 & C. wuellerstorfi & +3.10 & +3.74 & +0.73 & +0.73 \\
\hline $4-4$ & $44-46$ & 33.75 & C. wuellerstorfi & +2.96 & +3.60 & +0.34 & +0.34 \\
\hline $4-6$ & $54-56$ & 36.85 & C. wuellerstorfi & +3.10 & +3.74 & +0.73 & +0.73 \\
\hline $5-2$ & $44-46$ & 40.35 & Cibicides sp. & +3.53 & +4.17 & -0.28 & -0.28 \\
\hline $5-2$ & $44-46$ & 40.35 & $C$. teretis & $\begin{array}{r}3.58 \\
+3.58\end{array}$ & +3.88 & -1.49 & -0.24 \\
\hline $5-4$ & $44-46$ & 44.05 & C. wuellerstorfi & +2.61 & +3.25 & +0.33 & +0.33 \\
\hline $5-6$ & $44-46$ & 46.35 & P. murrhina & +3.56 & +3.56 & +0.80 & +0.80 \\
\hline
\end{tabular}

Table 5. Oxygen and carbon isotopic composition of planktonic foraminifers at Hole 610.

\begin{tabular}{|c|c|c|c|c|c|}
\hline $\begin{array}{l}\text { Core- } \\
\text { section }\end{array}$ & $\begin{array}{c}\text { Sample } \\
\text { interval } \\
(\mathrm{cm})\end{array}$ & $\begin{array}{l}\text { Sub-bottom } \\
\text { depth } \\
\text { (m) }\end{array}$ & Species & $\delta^{18} \mathrm{O}_{\mathrm{PDB}}$ & $\delta^{13} \mathrm{C}_{\mathrm{PDB}}$ \\
\hline $1-1$ & $44-46$ & 0.45 & N. pachyderma (L) & +3.94 & -0.32 \\
\hline $1-2$ & $44-46$ & 1.95 & N. pachyderma (L) & +3.43 & -0.39 \\
\hline $1-3$ & $44-46$ & 3.45 & N. pachyderma (L) & +2.59 & -0.53 \\
\hline $1-3$ & $44-46$ & 3.45 & N. pachyderma (R) & +1.27 & -0.45 \\
\hline $1-4$ & $44-46$ & 4.95 & N. pachyderma $(\mathrm{L})$ & +2.62 & +0.26 \\
\hline $1-4$ & $44-46$ & 4.95 & N. pachyderma (R) & +1.29 & -0.13 \\
\hline $1-5$ & $44-46$ & 6.45 & N. pachyderma (R) & +0.63 & -0.65 \\
\hline $1-6$ & $44-46$ & 7.95 & N. pachyderma $(\mathrm{L})$ & +3.71 & -0.27 \\
\hline $2-1$ & $44-46$ & 10.05 & N. pachyderma (L) & +3.59 & -0.36 \\
\hline $2-2$ & $44-46$ & 11.55 & N. pachyderma (R) & +0.91 & -0.26 \\
\hline $2-3$ & $44-46$ & 13.05 & N. pachyderma $(\mathrm{R})$ & +0.83 & -0.03 \\
\hline $2-4$ & $44-46$ & 14.55 & N. pachyderma (L) & +3.35 & -0.30 \\
\hline $2-5$ & $44-46$ & 16.05 & N. pachyderma $(\mathrm{L})$ & +3.41 & -0.43 \\
\hline 3-3 & $24-26$ & 22.45 & N. pachyderma $(\mathrm{R})$ & +0.59 & +0.21 \\
\hline $3-4$ & $44-46$ & 24.15 & N. pachyderma (L) & +3.45 & -0.19 \\
\hline $4-5$ & $44-46$ & 35.25 & N. pachyderma $(\mathrm{L})$ & +3.51 & -0.98 \\
\hline $4-6$ & $44-46$ & 36.85 & N. pachyderma (L) & +3.04 & -1.53 \\
\hline 4-6 & 5456 & 36.85 & N. pachyderma (R) & +0.84 & -0.75 \\
\hline $5-2$ & $44-46$ & 40.35 & N. pachyderma $(\mathrm{L})$ & +2.98 & -1.29 \\
\hline $5-5$ & $44-46$ & 44.85 & N. pachyderma $(\mathrm{L})$ & +3.16 & -0.41 \\
\hline $5-6$ & $44-46$ & 46.35 & N. pachyderma (L) & +2.42 & -0.42 \\
\hline
\end{tabular}

after, showing a generally smaller average terrestrial ice volume and concomittantly higher eustatic sea level for the early Quaternary/latest Pliocene. The standard deviation is also lower in the section prior to $0.9 \mathrm{Ma}$, indicating less severe climatic fluctuations in this part. In a study of the Quaternary section of Hole 502B, Prell (1982) identified a similar pattern showing two modes of climatic variability through the Quaternary. He proposed that the change of modes occurred at about $0.73 \mathrm{Ma}$, beneath the B/M boundary. Our results seem to indicate the presence of high-amplitude peaks down to approximately $0.9 \mathrm{Ma}$, but the exact timing of this change will have to be further validated by more detailed isotope records. The isotope records of Site 504 (Shackleton and Hall, 1983) and Site 552 (Shackleton et al., 1984) may also indicate less severe climatic oscillations in a period before $1 \mathrm{Ma}$. Hole 552A seems to indicate a return to larger amplitudes at about $2 \mathrm{Ma}$, continuing down to the first indication of large Northern Hemisphere glaciations at $2.4 \mathrm{Ma}$.

Hole 552A was located on the flank of the Rockall Bank not far to the west of our Site 610 (Fig. 1). At Hole $610 \mathrm{~A}$ we note evidence of larger amplitudes in the lowermost samples, at about $2.3 \mathrm{Ma}$, possibly correlative with similar features in the record from Hole 552A. Evidence from marine terraces in New Zealand indicates that there was an increase in amplitude of eustatic sealevel fluctuations at about $1 \mathrm{Ma}$ (Beu and Edwards, 1984), a conclusion in agreement with assumptions on sea-level changes based on oxygen isotope records.

The first input of ice-rafted detritus into the North Atlantic at Hole 552A has been rather accurately dated at $2.4 \mathrm{Ma}$ by nannofossil zonation (Shackleton et al., 1984). Blanc et al. (1983) also found that the first appearances of polar foraminiferal assemblages and icerafted detritus took place at about $2.5 \mathrm{Ma}$ at Site 116, located in the Rockall Basin area (Fig. 1). We record normal $N$. pachyderma $(\mathrm{L})$ in the lowermost samples from Hole $610 \mathrm{~A}$, but have not found this species in samples between this level (approximately $2.3 \mathrm{Ma}$ ) and a level slightly beneath the Jaramillo Event (approximately 1 Ma). With the caution that polar assemblages might be present as high-frequency peaks not detected by our coarse sample spacing, we suggest that this indicates: (1) Oceanic polar front oscillations across the Northeast Atlantic, typical of late Quaternary ocean circulation (Ruddiman and McIntyre, 1976), were generally not characteristic of the period between 1 and $2 \mathrm{Ma}$. This may imply that the role of the North Atlantic Ocean in climatic change in the late Quaternary, described by Ruddiman and McIntyre (1984), could have been significantly different from that in the early Quaternary. (2) The first part of the period recording Northern Hemisphere glaciations (approximately 2-2.5 Ma) might, as indicated by 


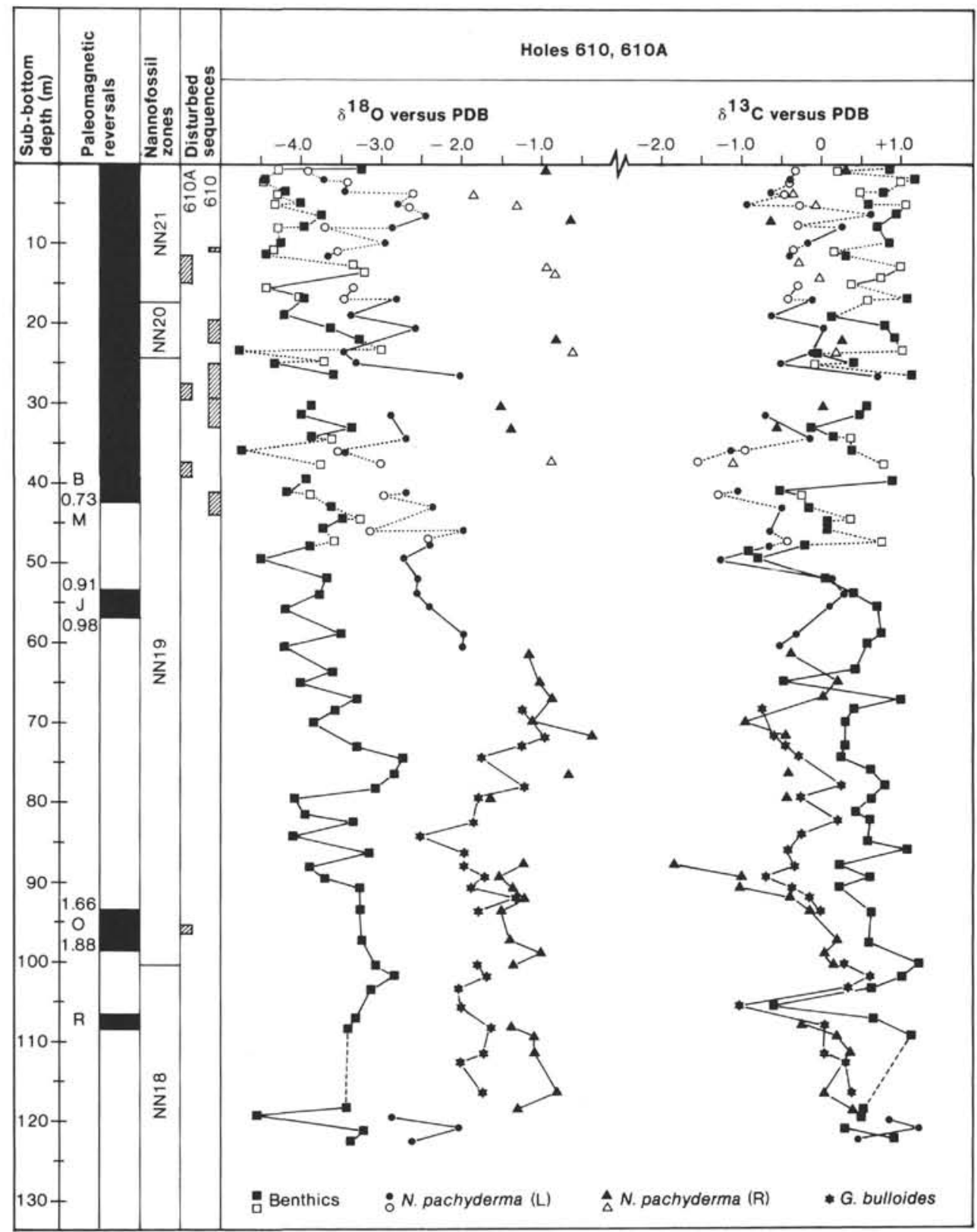

Figure 2. Oxygen and carbon isotope record from Holes 610 and 610A. (Open symbols indicate samples from 610, filled symbols indicate samples from 610A; paleomagnetic and nannofossil boundaries after Site 610 report [this volume]; $\mathbf{B}=$ Brunhes, $\mathbf{M}=$ Matuyama, $\mathrm{J}=$ Jaramillo, $\mathrm{O}=$ Olduvai, and $\mathbf{R}=$ Reunion.)

Table 6. Mean $(\chi)$ and standard deviation $(\sigma)$ of early and late Quaternary isotope records.

\begin{tabular}{|c|c|c|}
\hline & $\begin{array}{l}\text { late Quaternary } \\
\text { (0-0.9 Ma) }\end{array}$ & $\begin{array}{c}\text { early Quaternary/ } \\
\text { latest Pliocene } \\
(0.9-2.38 \mathrm{Ma})\end{array}$ \\
\hline$\delta^{18} \mathrm{O}$ benthic & $3.90 \% 0$ & $3.50 \%$ \\
\hline \multirow{2}{*}{${ }_{\delta}^{13} \mathrm{C}$ benthic } & 0.47 & 0.41 \\
\hline & $0.43 \%$ & $0.46 \%$ \\
\hline \multirow{2}{*}{${ }_{\delta}^{18} \mathrm{O}$ planktonic ${ }^{\sigma}$} & 0.51 & 0.52 \\
\hline & $2.53 \%$ & $1.57 \%$ \\
\hline \multirow{3}{*}{$\delta^{13} \mathrm{C}$ planktonic } & 0.98 & 0.53 \\
\hline & $-0.38 \%$ & $-0.10 \%$ \\
\hline & 0.47 & 0.55 \\
\hline
\end{tabular}

isotope variability and planktonic foraminifers, be characterized by large climatic oscillations and polar front migrations into the North Atlantic, analogous with the late Quaternary record.

\section{Carbon Isotopes}

Recent studies have documented the importance of benthic carbon isotope variations as an indicator of variations in the rates of deep-water renewal (Kroopnick, 1980; Shackleton et al., 1983; Duplessy et al., 1984). Foraminiferal evidence (Streeter and Shackleton, 1979; Streeter et al., 1982; Jansen et al., 1983) and carbon isotope variations (Duplessy, 1982; Curry and Lohmann, 1982; 


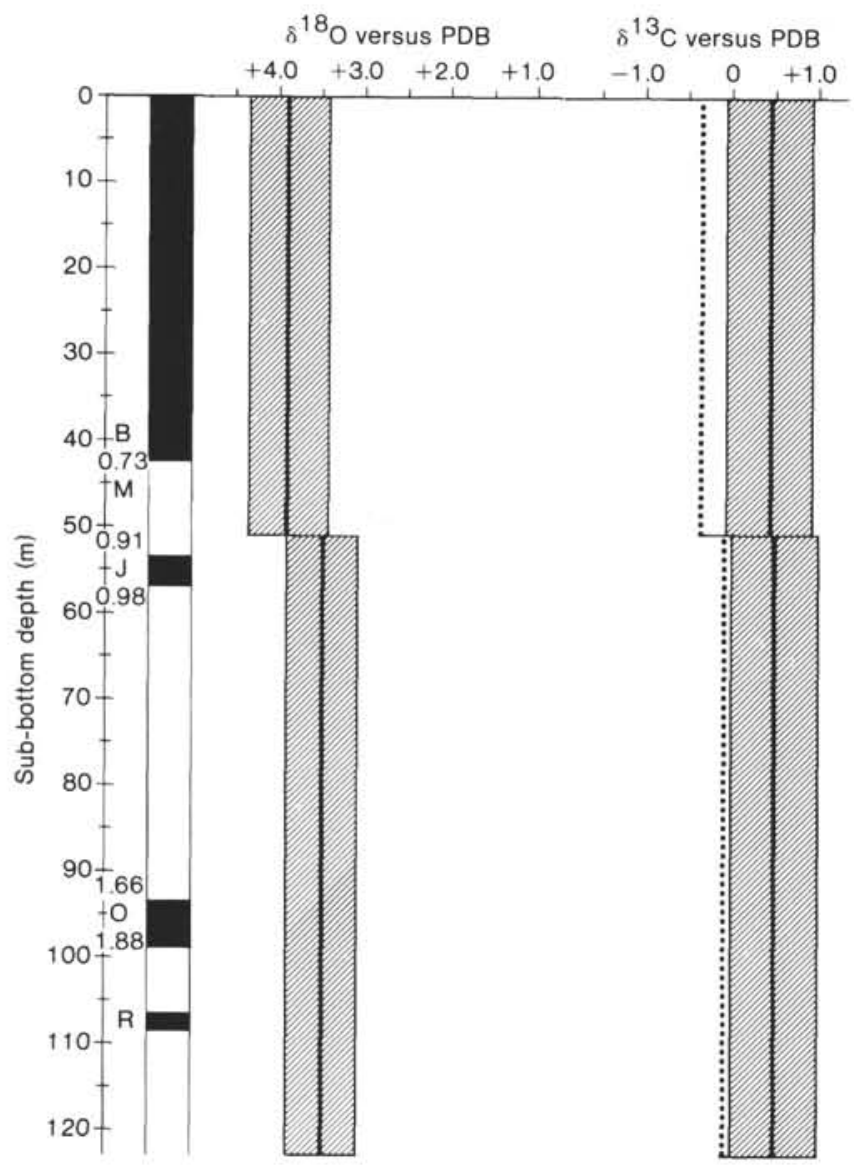

Figure 3. Mean carbon and oxygen isotope values in benthic foraminifers from Holes 610 and 610A (heavy line), standard deviation around the mean (light line and hachured area), and planktonic carbon isotopic mean (dotted line). Paleomagnetic boundaries after Site 610 report (this volume); $\mathrm{B}=$ Brunhes, $\mathrm{M}=$ Matuyama, $\mathrm{J}$

$=$ Jaramillo, $\mathrm{O}=$ Olduvai, and $\mathrm{R}=$ Reunion.

Shackleton et al., 1983) indicate large variations in the production rates of NADW and Norwegian-Greenland Sea Overflow in response to Quaternary climatic variations, with an assumed reduction in the production rates of NADW and hence in deep-water $\delta^{13} \mathrm{C}$ during glacials. As can be seen in Figure 2 and 3, glacial stages on the $\delta^{13} \mathrm{C}$ record do not display any notable change in mode in the mid-Quaternary, as did the $\delta^{18} \mathrm{O}$ record (Figs. 2 and 3, Table 6). This could imply that, despite reduced climatic variability in the early Quaternary, the variations in the production rates of NADW remained relatively constant in magnitude. Hence the $\delta^{13} \mathrm{C}$ variations imply that the on-off situation in Northern Hemisphere bottom-water formation was a feature in operation throughout the last $2.3 \mathrm{Ma}$ either as Norwegian-Greenland Sea Overflows or as deep-water formation in the North Atlantic itself.

In a study of benthic foraminiferal assemblages in cores from the Rio Grande Rise, Peterson and Lohmann (1982) proposed that a major change in South Atlantic deep-water circulation took place about 700 kyr. ago. The inflow of Antarctic Bottom Water (AABW) was reduced before this time, whereas the production of NADW did not seem to be affected. This contention seems to be in agreement with the carbon isotope record of Hole $610 \mathrm{~A}$, which does not indicate major changes in NADW variability across the time level of $0.7 \mathrm{Ma}$ (Figs. 2 and 3).

The mean benthic $\delta^{13} \mathrm{C}$ of $+0.45 \%$ at Hole $610 \mathrm{~A}$ confirms the conclusion of Shackleton et al. (1984) that the deep waters of the North Atlantic generally have been better ventilated than Pacific deep waters throughout the late Pliocene/Quaternary. The variability of the $\delta^{13} \mathrm{C}$ record at Hole 610A is similar to that found at other North Atlantic holes (Hole 552A-Shackleton et al., 1984; Hole $397-$ Shackleton and Cita, 1979). The mean $\delta^{13} \mathrm{C}$ value is close to that of Hole 552A, whereas the values for Site 397 (corrected for the difference in isotopic disequilibrium between Cibicides and Uvigerina) indicate lower $\delta^{13} \mathrm{C}$ levels for the deep water off West Africa. The difference might reflect the difference in deep-water residence time between the two regions, but may also in part reflect differences in surface productivity and in the input of organic matter to the bottom.

The planktonic $\delta^{13} \mathrm{C}$ record indicates variations of the same order of magnitude as the benthic record. The results for $N$. pachyderma $(\mathrm{L})$ duplicate some of the trends in the benthic curve (Fig. 2). The low sampling density, however, does not enable us to investigate further the correlation between near-surface and bottom $\delta^{13} \mathrm{C}$ response. In an area such as the North Atlantic, with numerous rapid changes in surface water masses and productivity, it is natural to expect large variations in planktonic $\delta^{13} \mathrm{C}$. Generally the planktonic $\delta^{13} \mathrm{C}$ values are lighter than the benthic values. More studies of vital effects, habitat, and feeding conditions of the species analyzed will be needed in order to evaluate the implications of this difference, but it might be suggested that the depletion reflects low $\delta^{13} \mathrm{C}$ levels in the productive upper water masses.

We have also compared mean benthic and planktonic $\delta^{13} \mathrm{C}$ before and after $0.9 \mathrm{Ma}$ (Fig. 3). The difference between benthic and planktonic $\delta^{13} \mathrm{C}$ values appears to decrease before $0.9 \mathrm{Ma}$, but this might be a result of the use of another planktonic species in the lower part of the record (Table 3 ).

\section{AMINO-ACID DIAGENESIS-RESULTS AND DISCUSSION}

The isoleucine epimerization in the total fraction (free + peptide bound) in $N$. pachyderma $(\mathrm{L})$ is presented as alle/Ile ratios in Table 7 and Figure 4. In the following discussion we assume a constant sedimentation rate of $5.2 \mathrm{~cm} / \mathrm{kyr}$. Down to about $20 \mathrm{~m}$ sub-bottom depth the alle/Ile ratios increase rather linearly to a ratio close to 0.3 . Further downcore we are able to register an increase in the alle/Ile ratio, but the reaction rate appears to be much lower. Similar trends of isoleucine epimerization have been demonstrated earlier for other planktonic species such as Globigerionoides sacculifer and Globorotalia tumida (King and Neville, 1977) and Orbulina universa and Globorotalia tumida menardii (group) (Müller, 1984). The inflection point in our material occurs at a different alle/Ile ratio than has been reported for other species. This might be due to changes in the natural 
Table 7. alle/Ile ratios in $N$. pachyderma $(\mathrm{L})$ from Site 610 .

\begin{tabular}{llcl}
\hline $\begin{array}{c}\text { Sample } \\
\text { (core-section, } \\
\text { interval in cm) }\end{array}$ & Lab no. & $\begin{array}{c}\text { Sub-bottom } \\
\text { depth } \\
(\mathrm{m})\end{array}$ & alle/lle \\
\hline $610-1-1,44-46$ & BAL 422 & 0.70 & 0.065 \\
$610-1-3,44-46$ & BAL 421 & 3.70 & 0.038 \\
$610 \mathrm{~A}-1-4,54-56$ & BAL 423 & 5.05 & 0.068 \\
$610 \mathrm{~A}-1-4,54-56$ & BAL 425 & 5.05 & 0.069 \\
$610 \mathrm{~A}-2-1,84-86$ & BAL 424 & 9.85 & 0.165 \\
$610-2-1,44-46$ & BAL 445 & 10.65 & 0.227 \\
$610-2-5,44-46$ & BAL 446 & 16.80 & 0.315 \\
$610 \mathrm{~A}-3-1,44-46$ & BAL 447 & 17.15 & 0.287 \\
$610 \mathrm{~A}-3-6,44-46$ & BAL 448 & 26.55 & 0.362 \\
$610 \mathrm{~A}-5-6,44-46$ & BAL 452 & 45.75 & 0.410 \\
$610 \mathrm{~A}-7-3,44-46$ & BAL 455 & 60.45 & 0.492 \\
$610 \mathrm{~A}-13-5,44-46$ & BAL 544 & 121.05 & 0.850 \\
$610 \mathrm{~A}-13-6,44-46$ & BAL 545 & 122.55 & 0.782 \\
\hline
\end{tabular}

hydrolysis reaction or leaching of isoleucine in the free fraction (Müller, 1984). In order to try to resolve this we plan to perform parallel analyses of the free fraction and more closely study hydrolysis reactions.

Earlier investigations of the isoleucine epimerization in foraminifers from the deep sea have mostly been based on material from piston cores from areas with low sedimentation rates (King and Neville, 1977; Müller, 1984). Our data might be more influenced by geothermal heat flow than these, especially in the lower part of the hole. To quantify this, more analyses are needed as well as comparisons with data on $N$. pachyderma $(\mathrm{L})$ from areas with low sedimentation rates.

King (1980), demonstrated that planktonic foraminiferal species could be divided into two different groups based on the overall composition of the protein. These groups parallel the taxonomic distinction between spinose and nonspinose forms. It is important to note that $N$. pachyderma's epimerization picture is more similar to the spinose $O$. universa than the nonspinose $G$. menardii tumida (group), as reported by Müller (1984).

Although we are only able to report preliminary results; it should be possible to draw some conclusions on the feasibility of using isoleucine epimerization in $N$. pachyderma as a geochronological tool in this type of material. The relatively steep curve back to approximately $0.3 \mathrm{Ma}$ indicates that the best possibility for using this method for absolute dating of deep-sea sediments is for material younger than $0.3 \mathrm{~m}$.y. The method might be of importance for older material, but probably with lower precision. An accurate kinetic model for isoleucine epimerization in $N$. pachyderma $(\mathrm{L})$ is needed, as this is a key taxon in many deep-sea records from high latitudes, and epimerization ratios have been used to raise questions concerning the difficult problem of the chronology of Arctic Ocean cores (Sejrup et al., 1984).

\section{CONCLUSIONS}

1. It should be feasible to produce a detailed stable isotope stratigraphy from both benthic and planktonic foraminifers through the upper Pliocene/Quaternary in Holes 610 and $610 \mathrm{~A}$. The high deposition rates at this site give a unique opportunity for providing a record that shows details in climatic response throughout this period.

2. The benthic oxygen isotope record indicates less climatic variability before $0.9 \mathrm{Ma}$ than after, with reduced amplitudes in isotopic fluctuations and generally smaller continental ice volume during the early Quaternary than in the late Quaternary.

3. Frequent oscillations of the oceanic polar front across the Northeast Atlantic seem to be dramatically reduced in the period between 2 and $1 \mathrm{Ma}$, as compared with the late Quaternary.

4. During the period between 2.5 and approximately 2.0 Ma, there may have been larger ice-volume fluctuations and polar-front oscillations than in the early Quaternary/latest Pliocene.

5 . The production rates of NADW as indicated by $\delta^{13} \mathrm{C}$ values were variable throughout the studied period, but the variance of NADW production remained unchanged throughout the Quaternary, unlike the $\delta^{18} \mathrm{O}$ record.

6. The extent of isoleucine epimerization in N. pachyderma $(\mathrm{L})$ suggest that amino-acid diagenesis has its greatest resolution as a geochronological tool over the last $300 \mathrm{kyr}$. Before this age reaction rate was reduced and the data show greater scatter.

\section{ACKNOWLEDGMENTS}

We thank Ida Beyer, Truls Johannessen, Karl-Johan Karlsen, and Kjell S $\phi$ gnen for assistance with sample processing and Jane Ellingsen for making the drawings. We also gratefully thank Sturla Rolfsen for his skilled efforts in keeping the mass-spectrometer operative, Julie Brigham-Grette for performing the amino-acid analyses and for her constructive suggestions concerning the manuscript, and Scott Lehman for carefully reading the manuscript, which helped us to improve it. Our reviewers Giff Miller and Warren Prell suggested many valuable improvements. The National Stable Isotope Laboratory at Bergen University is supported by grants from the Norwegian Research Council for Science and the Humanities (NAVF), which also supported this research through the program "Correlation of Marine and Continental Quaternary Stratigraphy.”

\section{REFERENCES}

Bada, J. L., and Man, E. A., 1980. Amino acid diagenesis in deep sea drilling project cores: kinetics and mechanisms of some reactions and their application in geochronology and in paleotemperature and heat flow determinations. Earth Sci. Rev., 16:21-55.

Belanger, P. E., Curry, W. B., and Mathews, R. K., 1981. Core-top evaluation of benthic foraminiferal isotopic ratios for paleo-oceanographic interpretations. Paleogeogr. Paleoclimatol. Paleoecol., 33: 205-220.

Beu, A. G., and Edwards, A. R., 1984. New Zealand Pleistocene and Late Pliocene glacio-eustatic cycles. Paleogeogr. Paleoclimatol. Paleoecol., 46:19-142.

Blanc, P. L., Fontugne, M. R., and Duplessy, J. C., 1983. The timetransgressive initiation of boreal ice-caps: continental and oceanic evidence reconciled. Paleogeogr. Paleoclimatol. Paleoecol., 43: 211-224.

CLIMAP Project Members, 1984. The last interglacial ocean. Quat. Res., 21:123-224.

Curry, W. B., and Lohmann, G. P., 1982. Carbon isotopic changes in benthic foraminifera from the western South Atlantic: reconstructions of glacial abyssal circulation patterns. Quat. Res., 18:218235.

Duplessy, J. C., 1982. Circulation des eaux profondes Nord Atlantiques au cours du dernier cycle climatique. Bull. Inst. Bass. d'Aquitaine, 31:379-391. 
Duplessy, J. C., Delibrias, G., Turon, J. L., Pujol, C., and Duprat, J., 1981. Deglacial warming of the northeastern North Atlantic Ocean: correlation with the paleoclimatic evolution of the European continent. Paleogeogr. Paleoclimatol. Paleoecol., 35:121-144.

Duplessy, J. C., Moyes, J., and Pujol, C., 1980. Deep-water formation in the North Atlantic Ocean during the last ice-age. Nature, 186:479-482.

Duplessy, J. C., Shackleton, N. J., Mathews, R. K., Prell, W. L., Ruddiman, W. R., Caralp, M., and Hendy, C. H., $1984 .{ }^{13} \mathrm{C}$ record of benthic foraminifera in the last interglacial ocean: implications for the carbon cycle and the global deep water circulation. Quat. Res., $21: 225-243$.

Ganssen, G., 1983. Dokumentation von küstennahem Auftrieb anhand stabiler isotopen in rezenten Foraminiferen von Nordwest-Afrika. "Meteor" Forsch. Ergebnisse, 37:1-46.

Graham, D. W., Corliss, B. H., Bender, M. L., and Keigwin, L. D., 1981. Carbon and oxygen isotopic disequilibria of recent deep-sea benthic foraminifera. Mar. Micropaleontol., 6:483-497.

Jansen, E., Sejrup, H.-P., Fjaeran, T., Hald, M., Holtedahl, H., and Skarb $\phi$, O., 1983. Late Weichselian paleoceanography of the southeastern Norwegian Sea. Norsk Geol. Tidsskr., 63:117-146.

Kellogg, T. B., Duplessy, J. C., and Shackleton, N. J., 1978. Planktonic foraminiferal and oxygen isotope stratigraphy and paleoclimatology of Norwegian Sea deep-sea cores. Boreas, 7:61-73.

King, K., Jr., 1980. Applications of amino acid epimerization for dating marine sediments. In Hare, P. E., Hoering, T. C., and King, K., Jr. (Eds.), Biogeochemistry of Amino Acids: New York (J. Wiley), pp. 377-391.

King, K., Jr., and Neville, C., 1977. Isoleucine epimerization for dating marine sediments: importance of analyzing monospecific foraminiferal samples. Science, 195:1333-1335.

Kroopnick, P., 1980. The distribution of ${ }^{13} \mathrm{C}$ in the Atlantic Ocean. Earth Planet. Sci. Lett., 49:211-217.

Miller, G. H., Sejrup, H.-P., Mangerud, J., and Andersen, B. G., 1983. Amino acid ratios in Quaternary molluscs and foraminifera from western Norway: correlation, geochronology and paleotemperature estimates. Boreas, 12:107-124.

Müller, P. J., 1984. Isoleucine epimerization on Quaternary planktonic foraminifera: effects of diagenetic hydrolysis and leaching, and Atlantic-Pacific intercore correlations. "Meteor" Forsch. Ergebnisse, 38:25-47.

Peterson, L. C., and Lohmann, G. P., 1982. Major change in Atlantic deep and bottom waters $700,000 \mathrm{yr}$. ago: benthonic foraminiferal evidence from the South Atlantic. Quat. Res., 17:26-38.

Prell, W., 1982. Oxygen and carbon isotope stratigraphy for the Quaternary of Hole 502B: evidence for two modes of isotopic variability. In Prell, W. L., Gardner, J. V., et al., Init. Repts. DSDP, 68: Washington (U.S. Govt. Printing Office), 455-464.

Ruddiman, W. F., and McIntyre, A., 1976. Northeast Atlantic paleoclimatic changes over the past 600,000 years. In Cline, R. M., and Hays, J. D. (Eds.), Investigation of late Quaternary paleoceanography and paleoclimatology. Geol. Soc. Am. Mem., 145:111-146.
1984. An evaluation of ocean-climate theories on the North Atlantic. In Berger, A. L., Imbrie, J., Hays, J., Kukla, G., and Salzman, B. (Eds.), Milankowitch and Climate, Part 2: Dordrecht (D. Reidel Publishing Co.), pp. 671-686.

Sejrup, H.-P., Miller, G. H., Brigham-Grette, J., L $\phi$ vlie, R., and Hopkins, D., 1984. Amino acid epimerization implies rapid sedimentation rates in Arctic Ocean cores. Nature, 310:772-775.

Shackleton, N. J., 1967. Oxygen isotope analyses and paleotemperatures reassessed. Nature, 215:15-17.

1974. Attainment of isotopic equilibrium between ocean water and the benthonic foraminifera genus Uvigerina: isotopic changes in the ocean during the last glacial. Colloques Internationaux CNRS, 219:203-209.

Shackleton, N. J., Backman, J., Zimmerman, H., Kent, D. V., Hall, M. A., Roberts, D. G., et al., 1984. Oxygen isotope calibration of the onset of ice-rafting and history of glaciation in the North Atlantic region. Nature, 307:620-623.

Shackleton, N. J., and Cita, M. B., 1979. Oxygen and carbon isotope stratigraphy of benthic foraminifers at Site 397: detailed history of climatic change during the late Neogene. In von Rad, U., Ryan, W. B. F., et al., Init. Repts. DSDP, 47, Pt. 1: Washington (U.S. Govt. Printing Office), 433-445.

Shackleton, N. J., and Hall., M. A., 1983. Stable isotope record of Hole 504 sediments: high resolution record of the Pleistocene In Cann, J. R., Langseth, M. G., Honnorez, J., Von Herzen, R. P., White, S. M., et al., Init. Repts. DSDP, 69: Washington (U.S. Govt. Printing Office), 431-441.

Shackleton, N. J., Imbrie, J., and Hall, M. A., 1983. Oxygen and carbon isotope record of East Pacific Core V19-30: implications for the formation of deep-water in the late Pleistocene North Atlantic. Earth Planet. Sci. Lett., 65:233-244.

Shackleton, N. J., and Opdyke, N. D., 1973. Oxygen isotope and paleomagnetic stratigraphy of equatorial Pacific Core V28-238: oxygen isotope temperatures and ice volumes on a $10^{5}$ year and $10^{6}$ year scale. Quat. Res., 3:39-55.

Streeter, S. S., Belanger, P. E., Kellogg, T. B., and Duplessy, J. C., 1982. Late Pleistocene paleoceanography of the Norwegian-Greenland Sea: benthic foraminiferal evidence. Quat. Res., 18:72-120.

Streeter, S. S., and Shackleton, N. J., 1979. Paleocirculation of the deep North Atlantic: a 150,000 year record of benthic foraminifera and $\delta^{18} \mathrm{O}$. Science, 203:168-171.

Wehmiller, J. F., 1984. Interlaboratory comparison of amino acid enantiomeric ratios in fossil Pleistocene molluscs. Quat. Res., 22: 109-120.

Woodruff, F., Savin, S. M., and Douglas, R. G., 1980. Biological fractionation of oxygen and carbon isotopes by Recent benthic foraminifera. Mar. Micropaleontol., 5:3-11.

Date of Initial Receipt: 9 November 1984

Date of Acceptance: 28 June 1985 
E. JANSEN, H. P. SEJRUP

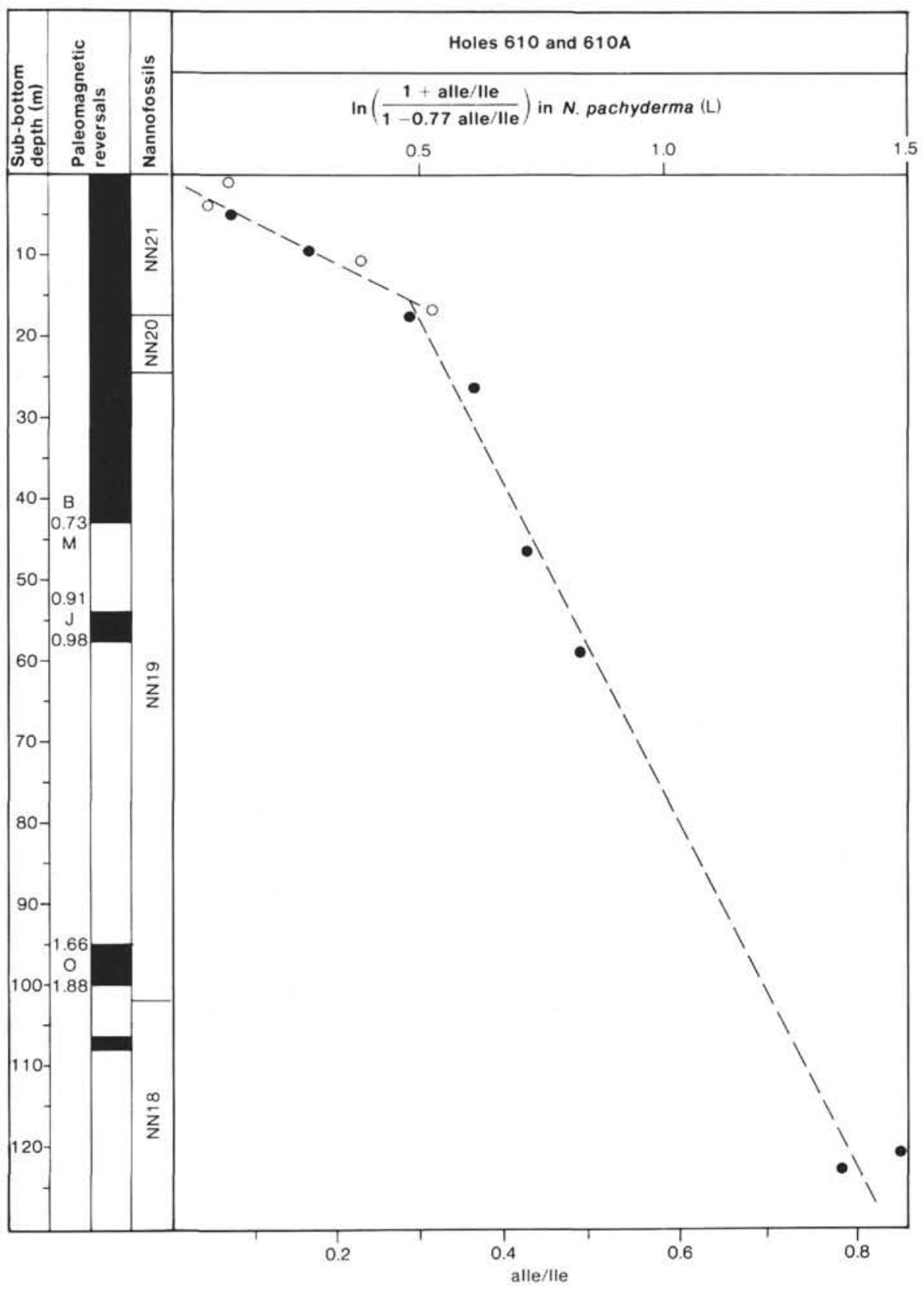

Figure 4. The alle/Ile ratios in N. pachyderma (L) from Holes 610 (open circles) and Hole 610A (closed circles). Paleomagnetic and nannofossil boundaries after Site 610 report (this volume). B $=$ Brunhes, $\mathrm{M}=$ Matuyama, $\mathrm{J}=$ Jaramillo, and $\mathrm{O}=$ Olduvai. 